\title{
The StarT back screening tool and a pain mannequin improve triage in individuals with low back pain at risk of a worse prognosis - a population based cohort study
}

Emma Haglund ${ }^{1,2^{*}}$ (D), Ann Bremander $2,3,4,5$ and Stefan Bergman ${ }^{2,3,6}$

\begin{abstract}
Background: The STarT Back Screening Tool (SBT) identifies patients with low back pain (LBP) at risk of a worse prognosis of persistent disabling back pain, and thereby facilitates triage to appropriate treatment level. However, the SBT does not consider the pain distribution, which is a known predictor of chronic widespread pain (CWP). The aim of this study was to determine if screening by the SBT and screening of multisite chronic widespread pain (MS-CWP) could identity individuals with a worse prognosis. A secondary aim was to analyze self-reported health in individuals with and without $L B P$, in relation to the combination of these two screening tools.

Methods: One hundred and nineteen individuals (aged 40-71 years, mean (SD) 59 (8) years), 52 with LBP and 67 references, answered two screening tools; the SBT and a pain mannequin - as well as a questionnaire addressing self-reported health. The SBT stratifies into low, medium or high risk of a worse prognosis. The pain mannequin stratifies into either presence or absence of CWP in combination with $\geq 7$ painful areas of pain (0-18), here defined as MS-CWP (high risk of worse prognosis). The two screening tools were studied one-by-one, and as a combined screening. For statistical analyses, independent t-tests and Chi-square tests were used.

Results: Both the SBT and the pain mannequin identified risk of a worse prognosis in individuals with ( $p=0.007)$ or without ( $p=0.001$ ) LBP. We found that the screening tools identified partly different individuals at risk. The SBT identified one individual, while the pain mannequin identified 21 (19\%). When combining the two screening methods, 21 individuals (17\%) were at high risk of a worse prognosis. When analyzing differences between individuals at high risk (combined SBT and MS-CWP) with those at low risk, individuals at high risk reported worse health $(p=0.013-<0.001)$.

Conclusions: Both screening tools identified individuals at risk, but they captured different aspects, and also different number of individuals at high risk of a worse prognosis. Thus, using a combination may improve early detection and facilitate triage to appropriate treatment level with multimodal approach also in those otherwise missed by the SBT.
\end{abstract}

Keywords: Low back pain, Chronic widespread pain, Multisite pain, Population-based cohort, Prognostic indicators, Questionnaire

\footnotetext{
* Correspondence: Emma.haglund@hh.se

${ }^{1}$ School of Business, Engineering and Science, Halmstad University, Halmstad,

Sweden

${ }^{2}$ Spenshult Research and Development Center, Halmstad, Sweden

Full list of author information is available at the end of the article
}

(c) The Author(s). 2019 Open Access This article is distributed under the terms of the Creative Commons Attribution 4.0 International License (http://creativecommons.org/licenses/by/4.0/), which permits unrestricted use, distribution, and reproduction in any medium, provided you give appropriate credit to the original author(s) and the source, provide a link to the Creative Commons license, and indicate if changes were made. The Creative Commons Public Domain Dedication waiver (http://creativecommons.org/publicdomain/zero/1.0/) applies to the data made available in this article, unless otherwise stated. 


\section{Background}

Low back pain (LBP) is one of the world's leading health problems [1-3]. The point prevalence is around $40 \%$ in the western world. The symptoms often cause activity limitations and participation restrictions, even though the condition is usually transient [4]. Primary health care is often the first point of care for patients with LBP. Most individuals with LBP have a condition defined as non-specific, in contrast to serious LBP pathology or nerve pathology [5]. Pain in general can be categorized as acute or chronic; it is considered chronic if the pain has been present for at least three months [6]. Besides the individual suffering, LBP can cause an economic burden to the individuals and society [2].

The prevalence of chronic LBP (CLBP) is estimated to affect approximately $23 \%$ of the population, but is a more complex condition [7, 8]. It is also known that individuals who once suffered with LBP have recurring episodes of LBP more often over the course of their lives than those who have never had LBP [9]. Knowledge of fundamental prognostic factors will help to understand an individual's risk of a poorer prognosis and chronic condition. Heavy physical work demands and low physical function are physical risk factors for developing CLBP [10]. Other factors of importance are older age, low general health, stress symptoms, radiating pain, high pain intensity, previous sick-leave due to LBP and low education level [10-12]. The biopsychosocial approach, in which underlying social and psychological factors have also been found to be important, may further increase the knowledge concerning CLBP. Low job satisfaction, low social support at work, sadness and depression are some of these risk factors [13-15]. Kinesiophobia, feelings of fear-avoidance of work activities, physical activities, and catastrophizing (imagining a situation worse than it is) are other factors that seems to influence risk of a worse prognosis and the development of CLBP [15-17]. There is also growing evidence of an association between LBP, concomitant pain in other regions of the body, and the risk of developing more generalized pain [18]. Widespread pain (axial, present in both sides of the body, in upper and lower limb), as well as multisite pain (seven or more anatomical sites), increases the risk of developing a chronic condition [19-21]. An overlap between multisite and chronic widespread pain (CWP) has also been shown [22].

Clinicians need easy-to-use instruments to identify different subgroups of patients with LBP, taking into account early prognostic factors of worse prognosis. The screening instrument STarT (Subgroups for Targeted Treatment) Back Screening Tool (SBT) is a short and easy-to-score tool that identifies individuals at risk of a worse prognosis in order to facilitate triage to appropriate treatment level [23]. The SBT score stratifies individuals to low, medium or high risk of a worse prognosis. Low risk implies management with general advice, medium risk evidence-based physiotherapy and the high-risk group cognitive behavioral therapy. The SBT takes into account known risk factors such as radiating pain, activity limitations, kinesiophobia and catastrophizing, and has shown predictive value for functional improvements two months after a visit to primary care [24]. When using the SBT for triaging to different risks and treatments in primary care settings, in comparison with a control group not using the SBT, the stratified approach has been shown to improve outcome measures such as pain intensity, catastrophizing, fear, anxiety, depression, general health and absenteeism [25]. However, the SBT does not capture all of those at high risk [26, 27]. Thus, the instrument may need supplemental information about the distribution of pain or if the pain is multisite or not.

The American College of Rheumatology's (ACR) definition of CWP [20] has been used in epidemiological studies to classify individuals with pain symptoms into subgroups [22]. The definition states that CWP is pain (i) for at least three months during the past 12, (ii) distributed in the axial skeleton, (iii) in both sides of the body, and (iv) in the upper and lower limbs. In a modification of the ACR's diagnostic criteria in pain syndromes such as fibromyalgia, the widespread pain index has been used to assess if patients have multisite pain or not. Seven or more regions of pain were considered as worse severity of fibromyalgia [21]. Pain mannequins have been used in epidemiological studies and are considered valid and reliable measures for the assessment of pain distribution $[28,29]$. Screening for physical and psychosocial risk factors together with pain related symptoms by using STB, and by adding the pain mannequin aim to catch important aspects associated to a worse prognosis of persistent disabling back pain [26,30].

The SBT does not consider pain distribution or if the pain is multisite or not. This study compared screening by the SBT with screening of multisite chronic widespread pain (MS-CWP) based on a pain mannequin, in a population-based group of individuals with and without back pain. The aim was to analyze to what extent the two screening tools could identify individuals with worse prognosis. A secondary aim was to analyze self-reported health in individuals with and without LBP, in relation to the combination of these two screening tools.

\section{Methods}

This study included a sub group of individuals from a well-established cohort (EPIPAIN) in south west Sweden $[19,29]$. EPIPAIN is a population-based longitudinal cohort study. At the start in 1995, EPIPAIN included 3928 individuals representative of the adult population, aged 
18-74 years. Since then, there have been four follow-ups (1998, 2003, 2007, 2016). The latest survey in May 2016 was sent to 1832 individuals, whereof $65 \%$ (1184) responded. This survey serves as the basis for inclusion in the current study. More details of the EPIPAIN project can be read elsewhere $[19,29]$. To answer the research questions the included individuals filled in a more comprehensive questionnaire in connection with a clinical visit that took place sometime in between August to November 2016.

\section{Subjects}

A total of 236 individuals aged 40 to 70 years responding to the 2016 EPIPAIN questionnaire were selected to the present study and invited to take part in clinical tests and to answer an additional questionnaire. This included all 176 individuals with a self-report of back pain for three months or more during the last 12 months. Sixty individuals from those reporting no chronic pain (NCP) during the last 12 months were randomly selected and also invited to the study. Of those invited, 126 individuals (83 with chronic LBP and 43 with $\mathrm{NCP}$ ) agreed to participate.

To have a current knowledge of the LBP status in connection to the clinical visit, three to six months after the EPIPAIN questionnaire, the individuals answered the question: Have you had low back pain during the last week? (answer yes/no). The answer to this question formed the two groups, the LBP and the reference group, that were used for the analyses in the study (Fig. 1). This assignment did not take their earlier report of chronic pain into consideration. The current report of chronic pain based on results from the questionnaire filled out in connection with the clinical visit were used for analyses in the study.

\section{Instruments}

\section{STarT back screening tool}

The questionnaire survey consisted of two screening tools, and several validated self-report instruments assessing the impact of physical and mental health. The primary screening tool was the SBT, classifying individuals into three different risk levels of worse prognosis (risk for persistent disabling back pain) in order to facilitate triage to appropriate treatment $[23,26]$. The SBT includes four questions concerning physical risk factors for worse prognosis: (i) referred leg pain, (ii) neck/shoulder pain, (iii) disability in walking, (iv) difficulties in dressing; and five questions concerning psychosocial factors: (i) fear, (ii) anxiety, (iii) catastrophizing, (iv) feelings of depression and (v) bothersomeness. The sum gives a total score of $0-9$ (best to worst) which sorts the

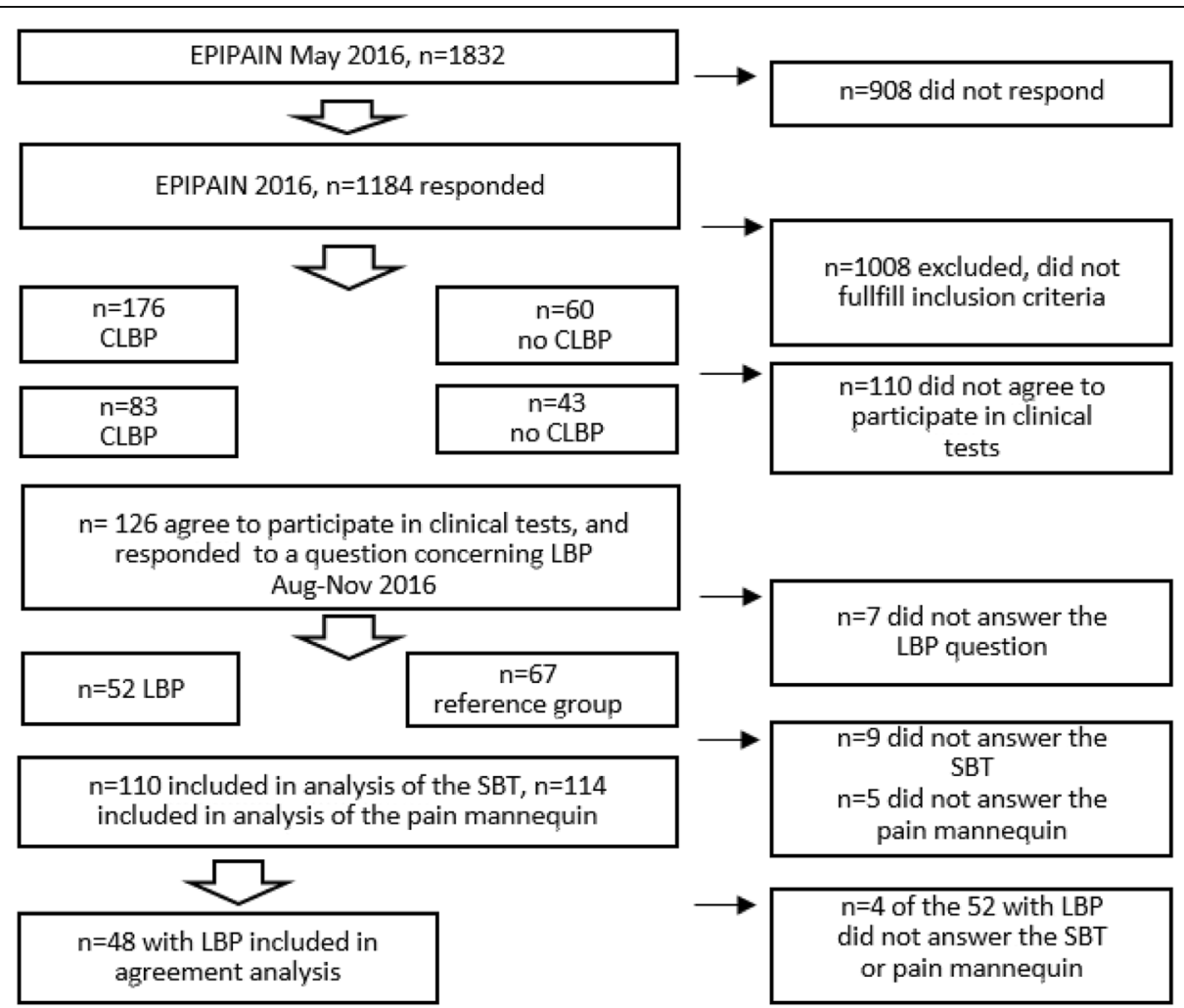

Fig. 1 Flow chart showing the participation in the study. EPIPAIN=Population-based longitudinal cohort study. CLBP=chronic low back pain; reports of pain for three months or more during the last 12 months and LBP according to the pain mannequin. STB=STarT Back Screening Tool 
individuals into low, medium or high risk of a worse prognosis $[23,26]$. The SBT has shown acceptable validity and reliability [31-34]. Low risk of a worse prognosis is defined if the overall tool score is $\leq 3$. The medium risk group included individuals with an overall score of $\geq 4$ in combination with psychosocial subscale score $<4$. The high-risk group is those individuals with a psychosocial subscale score $\geq 4$.

\section{Pain mannequin}

The second screening tool was a pain mannequin including questions about the experience and distribution of chronic musculoskeletal pain. The pain mannequin was developed within the EPIPAIN project, constructed in accordance with the ACR definition of CWP [20], and found to have good validity and reliability [35]. It has been presented in more detail in previous studies $[19,29]$. In the heading of the pain mannequin an explanation stated that the questions refer to aches and pains in joints and soft tissues. An initial question was used to consider if the pain was chronic or not: Have you experienced any aches or pain lasting more than three months during the past twelve months? according to ACR definition [20]. A mannequin with predefined body regions [29] followed the question to categorize individuals into CWP, chronic regional pain (CRP) or NCP. Individuals were categorized to CWP if they had experienced pain symptoms for at least three months during the past 12 , and reported widespread pain in the mannequin, that is pain in the axial skeleton, in both sides of the body and in upper and lower part of the body. If they had experienced pain symptoms for the same duration but did not report widespread distribution, they were categorized as CRP. Those with no pain experience for at least three months during the past 12 were categorized as NCP [20]. The number of predefined painful body regions (0-18) were also counted [29]. Presence of seven or more painful regions was designated as multisite pain (MS) [19]. A presence of CWP in combination with $\geq 7$ painful regions were designated as MS-CWP. MSCWP is known to be associated to a more persistent pain condition (worse prognosis) [19].

\section{Other questionnaires}

Pain intensity the previous week was recorded using a numeric rating scale (NRS), 0-10 (best to worst) [36]. Low back pain in relation to physical function or disability was assessed by the low back pain specific RolandMorris Disability Questionnaire (RMDQ), 0-24 (no disability to maximum disability) [37]. Self-reported health state was assessed by the generic EuroQol-5 domain questionnaire (EQ-5D), 0-1 (no health to full health) [38]. Mental health was assessed by the Hospital Anxiety (HAD-A) and Depression (HAD-D) scales, each of these subscales, scoring 0-21 (no distress to maximum distress) [39]. Kinesiophobia was assessed by the FearAvoidance Beliefs Questionnaire, evaluating to what extent physical activity (FABQ-PA, subscale one) and work (FABQ-Work, subscale two) affect the pain experience. The score ranged from 0 to 24 in the FABQ-PA and 0 to 42 in the FABQ-Work, (no fear to high scores of fear and avoidance behavior) [40, 41].

\section{Statistical analyses}

Descriptives of the sample are presented as mean and standard deviation (SD) or as frequencies (\%). Independent $\mathrm{t}$-tests or Chi-square were used to describe differences in self-reported health and risk screening in the two groups. Independent t-tests were used to analyze differences in self-reported physical and mental health between individuals with different risk levels based on the combination of the SBT and the pain mannequin. The combination implies that all individuals at high risk captured by the SBT or presence of MS-CWP by the pain mannequin identified as at high risk of a worse prognosis. Thus, individuals at low or medium risk assessed by the SBT, or those showing no MS-CWP, were stratified to low risk of a worse prognosis in the combined screening.

\section{Results}

Hundred twenty-six (53\%) of the 236 identified individuals accepted the invitation of a clinical visit. When filling in the EPIPAIN follow-up questionnaire in May 2016, 83 participants (61\% women) reported chronic low back pain, while 43 participants (56\% women) reported $\mathrm{NCP}$. In those who declined an invitation, men were more prevalent.

At the time for the clinical visit, 52 individuals reported LBP and 67 did not and formed the reference group. Seven did not answer the question about LBP in the last week, and thus 119 were available for analysis. The mean (SD) age of the sample $(n=119)$ was 59 (8) years, ranging between 41 and 71 years, and $75(60 \%)$ were women. There were no differences in age and sex between the two groups (Table 1). Individuals with LBP reported worse physical and mental health and a worse SBT score compared with individuals in the reference group. The LBP group also reported a larger number of painful regions and a higher frequency of CWP than the references (Table 1).

\section{Risk assessment by the different screening tools}

When stratifying individuals into low, medium or high risk of a worse prognosis by the SBT, 41 individuals $(80 \%)$ in the LBP group and 58 in the reference group (98\%) were stratified into the low risk group. Nine individuals (18\%) with LBP were stratified as medium risk and one as at high risk of a worse prognosis. There was 
Table 1 Self-reported health in individuals with low back pain vs reference group (individuals with no LBP)

\begin{tabular}{llll}
\hline & Low back pain $(n=52)$ & Reference group $(n=67)$ & $p$-value \\
\hline Age (years) & $60(7)$ & $59(9)$ & 0.233 \\
Sex (women) & $65 \%$ & $57 \%$ & 0.337 \\
SBT (0-9) & $2.4(1.5)$ & $0.7(0.9)$ & $<0.001$ \\
RMDQ (0-24) & $6.6(4.7)$ & $2.0(2.1)$ & $<0.001$ \\
EQ5D (0-1) & $0.69(0.21)$ & $0.87(0.14)$ & $<0.001$ \\
FABQ PA (0-24) & $8.4(5.9)$ & $5.5(5.4)$ & $<0.001$ \\
FABQ work (0-42) & $15.0(12.3)$ & $8.5(8.2)$ & $<0.001$ \\
HAD anxiety (0-21) & $7.7(2.9)$ & $5.1(3.4)$ & $<0.001$ \\
HAD depression (0-21) & $3.7(1.8)$ & $2.2(2.0)$ & $<0.001$ \\
Pain NRS (0-10) & $5.1(2.1)$ & $1.2(2.4)$ & $<0.001$ \\
Regions with pain (0-18) & $5.0(4.2)$ & $1.9(3.0)$ & $<0.001$ \\
CWP at inclusion & $53 \%$ & $18 \%$ & $<0.001$ \\
\hline
\end{tabular}

Presented as mean (SD) or frequencies and $p$-values. SBT = STarT Back Screening Tool (best-worst), RMDQ = Roland-Morris Disability Questionnaire (best-worst), $\mathrm{EQ} 5 \mathrm{D}=$ EuroQol 5-domain (worst-best), $\mathrm{FABQ}=$ Fear-Avoidance Beliefs Questionnaire for physical activity and work (best-worst), HAD = Hospital Anxiety and Depression scale (best-worst), NRS=Numeric Rating Scale, LBP = Low Back Pain, CWP = Chronic widespread pain, CLBP=Chronic LBP

a statistically significant difference in risk distribution based on the SBT between the LBP group and references, $p=0.007$ (Table 2). The pain mannequin showed a statistically significant difference in risk distribution between the LBP and the reference group, $p<0.001$. In the LBP group, 16 individuals (33\%) reported MS-CWP and were considered as at high risk of a worse prognosis. Four of the references (6\%) also reported MS-CWP, but did not report LBP last week (Table 2). Nine individuals did not complete the SBT and five did not complete the pain mannequin, thus these analyses were based on 110 and 114 individuals respectively.

Forty-eight individuals with LBP were available for analysis of the agreement between the two screening methods (SBT vs. pain mannequin). Only one individual was at high risk as captured by the SBT. Using the pain mannequin, 16 (33\%) had MS-CWP and were defined as at high risk of a worse prognosis. Eleven individuals (23\%) reported MS-CWP in the SBT low risk-group and

Table 2 Screening by the STarT Back Screening Tool (SBT) ( $n=$ $110)$ and by pain mannequin $(n=114)$

\begin{tabular}{lll}
\hline & Low back pain & Reference group \\
\hline SBT - Low risk & $41(80)$ & $58(98)$ \\
SBT - Medium risk & $9(18)$ & $1(2)$ \\
SBT - High risk & $1(2)$ & 0 \\
No MS-CWP & $33(67)$ & $61(94)$ \\
MS-CWP & $16(33)$ & $4(6)$ \\
\hline
\end{tabular}

Screening by the SBT for low, medium or high risk of a worse prognosis and by pain mannequin for multisite chronic widespread pain (MS-CWP) or not (No MS-CWP) in both groups (LBP vs. reference group). Presented as number of individuals and percent (\%). MS-CWP $=$ multisite pain in $\geq 7$ sites and chronic widespread pain distribution four individuals reported MS-CWP in the medium riskgroup (Table 3).

\section{Differences in self-reported health}

Sixteen individuals $(31 \%)$ were included in the high-risk group for a worse prognosis when the two screening tools were combined. Individuals at high risk reported worse health with statistically significant differences in reports of anxiety (HAD-A), depression (HAD-D), and pain intensity (NRS) when compared with those at low risk. The trend was also uniform, but not statistically significant, for their reports of physical function (RMDQ) and self-reported health (EQ5D) (Table 4).

\section{Discussion}

Back pain is a major health problem for society as well as one of the leading causes of disability (3). Thus, it is important in clinical practice to identify individuals at risk of worse prognosis early, and to develop a triage system for interventions identifying those in need of cognitive behavioral therapy. In this sub sample of a population-based cohort of individuals with and without self-reports of LBP, the risk of a worse prognosis was compared between the two different screening tools. Both the SBT and the pain mannequin discriminated between different risk levels of worse prognosis in individuals with or without LBP. A combination of the two screening tools seemed to also capture more individuals at risk of worse prognosis than if the SBT were used alone. Thus, the combination of the two screening tools may improve the ability to early detect those at risk of a worse prognosis and facilitate triage to appropriate treatment level in a clinical setting. 
Table 3 Observed agreement between the STarT Back Screening Tool and the pain mannequin, $n=48$

\begin{tabular}{llll}
\hline & \multicolumn{2}{l}{ STarT Back Screening Tool } & \\
\cline { 2 - 4 } & Low risk $(n=39)$ & Medium risk $(n=8)$ & High risk $(n=1)$ \\
\hline No MS-CWP $(n=32)^{*}$ & 28 & 4 & 0 \\
MS-CWP $(n=16)^{* *}$ & 11 & 4 & 1 \\
\hline
\end{tabular}

Analysis based on individuals with low back pain. Presented as number of individuals

${ }^{*}=$ No multisite chronic widespread pain (CWP). ${ }^{* *}=$ Multisite-CWP

\section{Risk assessment by the different screening tools}

We found significant differences in risk distribution between individuals with and without LBP for both the SBT and the pain mannequin. However, the SBT did not capture many at high risk $(<1 \%)$. In the original study where the SBT was developed, $15 \%$ were allocated to the highrisk group [23]. Other studies have found frequencies in the range of $0-32 \%$ at high risk of a worse prognosis when using the SBT [24, 25, 34, 42-44]. In an earlier study, noone at high risk was captured by the SBT, despite inclusion of health-care seeking patients [27]. We also found a higher frequency of individuals at low risk identified by the SBT (about 90\%) in comparison with earlier research where the reported frequencies of low risk individuals range from 26 to $66 \%$ [23-25, 27, 34, 42-44]. One reason for these dissimilarities could be explained by the different inclusion criteria and samples used in the different studies.

The SBT was primary developed, validated and proven as acceptable for use for individuals seeking primary health-care for their LBP [23, 34, 43], while this study used a population-based sample and the individuals were included regardless of the need for a health-care visit. To our knowledge, no other study has tested the SBT in a comparable setting of individuals from the general population. Therefore, it is not remarkable that our sample reported better health than described in earlier studies. For example, the mean RMDQ score for the total sample was found to be less than 5 in the current study, while others have reported a mean of 9 or higher [23-25, 43]. Also, the mean of pain intensity (score $>5)[25,27,34]$, and the SBT score (score 3-4) [15, 23, 27, 34, 42] were higher in earlier studies (compared with our 3.9 for pain and 1.8 for the SBT). Based on the SBT, most of the individuals were screened as at low risk, both individuals with LBP (80\%) and references (98\%). When combining the screening tools and studying self-reported health, the RMDQ almost reached a mean of 8 in those with high risk of a worse prognosis, which is more in line with earlier data. In the LBP group, $8 \%$ scored zero (lowest) in the SBT, while $53 \%$ of the reference group scored zero. None of the individuals scored 9 (the highest possible score) in the SBT in either of the groups. These results are in line with a recent study, where the comparable figures are $6 \%$ scoring 0 , and no-one scoring 9 [27].

In the current study, the SBT only identified one individual at high risk of worse prognosis, also captured when screening by the pain mannequin. Nearly onethird of those classified at low risk by the SBT and half of those classified as medium risk turned out to have had a simultaneous multisite chronic widespread pain (MS-CWP) by the pain mannequin. Multisite widespread pain has been found to influence the probability of worse prognosis and/or CLBP [30]. Even so, pain intensity or the distribution of pain are not considered in the nine questions of the SBT [23]. The risk of missing individuals with low SBT scores but high risk according to pain distribution, led to our hypothesis that a combined use of these screening tools would identify individuals at high risk of a worse prognosis more accurately. Conversely, half of those screened as at low risk by the pain mannequin were identified as medium risk by the

Table 4 Differences in self-reported physical or mental health, $n=52$

\begin{tabular}{|c|c|c|c|}
\hline & $\begin{array}{l}\text { Lower risk in combined screening }(n=36) \\
\text { Mean }(\mathrm{SD})\end{array}$ & $\begin{array}{l}\text { High risk in combined screening }(n=16) \\
\text { Mean (SD) }\end{array}$ & $p$-value \\
\hline RMDQ (0-24) & $5.8(4.1)$ & $7.8(5.3)$ & 0.228 \\
\hline EQ5D (0-1) & $0.72(0.18)$ & $0.63(0.27)$ & 0.155 \\
\hline FABQ PA (0-24) & $8.6(5.5)$ & $7.9(6.8)$ & 0.701 \\
\hline FABQ work (0-42) & $12.6(11.6)$ & $20.1(14.4)$ & 0.066 \\
\hline HAD anxiety (0-21) & $7.2(3.1)$ & $9.1(1.7)$ & 0.026 \\
\hline HAD depression (0-21) & $3.3(1.9)$ & $4.6(1.3)$ & 0.016 \\
\hline Pain NRS (1-10) & $4.5(2.0)$ & $6.3(1.9)$ & $<0.001$ \\
\hline
\end{tabular}

Analysis based on the triage by the combination of the SBT and pain mannequin in individuals with low back pain. SBT = STarT Back Screening Tool. Lower risk in combined screening $=$ SBT low and medium risk or no Multisite Chronic Widespread Pain (MS-CWP). High risk in combined screening = SBT high risk or MS-CWP. $\mathrm{RMDQ}=$ Roland-Morris Disability Questionnaire (best-worst), EQ5D = EuroQol 5-domain (worst-best), $\mathrm{FABQ}=$ Fear-Avoidance Beliefs Questionnaire for physical activity and work (best-worst), HAD = Hospital Anxiety and Depression scale (best-worst), NRS = numeric rating scale (best-worst) 
SBT. This clearly illustrates how the two instruments capture different aspects, and why a combination of the two might capture a more appropriate sample in need of cognitive behavioral therapy and multidisciplinary interventions.

\section{Differences in self-reported health}

We found a clear difference in self-reported health between individuals with LBP and those in the reference group, even if the result indicates that symptoms of pain are fluctuating slightly over time. The current study also found that, when combining the screening tools in order to allocate individuals into a low or high-risk of a worse prognosis, the low-risk group had significantly better self-reported physical and mental health concerning anxiety, depression, and pain intensity. There was also a trend towards differences in their reports of physical function, health-related quality of life and fear-avoidance. These important differences did not reach statistical significance, however, possibly due to a small sample size. In line with this, earlier research has described worse health in individuals with LBP than in the general population $[45,46]$ and those at low risk of a worse prognosis reported better health [24, 25, 27, 42, 44]. Other studies have shown that individuals with high levels of pain also had worse reported health [10, 47]. Thus, both screening tools used in the combined screening seemed to be able to identify different factors of worse prognosis. However, to use both instruments independently may lead to far too many being classified as at high risk of a worse prognosis. Thus, using a combination may improve the ability to capture also those with multisite widespread pain missed by the SBT.

\section{Strengths and limitations}

The observed agreement between the two screening tools and their ability to stratify individuals into different levels of risk of a worse prognosis (Table 3) clearly illustrates how the tools to some extent identify different individuals. Compared with using the SBT solely, the pain mannequin added individuals who otherwise would have been missed. An early identification of those at risk of a worse prognosis is of great value for both individuals and society, regardless of the underlying prognostic factor.

A limitation is that the analyses were based on a sample from the general population. It makes it somewhat difficult to compare the results with other studies that used the SBT in a clinical setting. Most studies testing the SBT used samples from health-seeking individuals in primary care, as the SBT was developed for that setting. However, not all individuals with LBP seek health care [8], and the heterogeneity of the current sample could well represent the general population and credible generalizations from this study can therefore be made.
Another limitation is the low number of individuals with a high risk of a worse prognosis based on the SBT. To better understand the benefits, the combination of these two screening tools needs to be tested in clinical practice in primary health care in a randomized controlled study. In future studies it is also important to consider if it is better to upgrade individuals identified at high risk by the pain mannequin if they have a medium risk of a worse prognosis by the SBT, otherwise a too large number of individuals may be triaged to treatment with cognitive behavioral therapy.

\section{Conclusion}

The SBT and the pain mannequin were complementary in identifying partly different individuals with LBP that could have a higher risk of a worse prognosis and are in need of a more comprehensive treatment. A combination of the two instruments may facilitate a triage to the appropriate treatment level, as individuals who are at high risk due to multisite chronic widespread pain are missed by the SBT, but picked up by the pain mannequin. This combined screening method needs to be tested in a clinical setting.

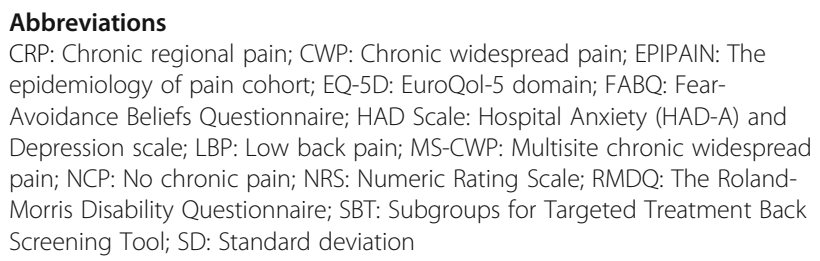

Acknowledgements

Not applicable.

\section{Authors' contributions}

All authors (EH; $A B ; S B$ ) designed and performed the study and revised and approved the final manuscript. EH and SB analyzed the data, while EH draft the manuscript.

\section{Funding}

The study was supported by grants from AFA insurance, the Swedish Rheumatism Association and Region Halland, Sweden. None of the funders had any influence on the study except for funding.

\section{Availability of data and materials}

The data from the current study are not publicly available according to the ethical approval and patients' informed consent, but could be available from the corresponding authors upon reasonable request.

\section{Ethics approval and consent to participate}

The study was approved by the Regional Ethical Review Board of Lund University, Sweden (entry no. 2016/325). Before filling in the questionnaires, individuals received oral and written information about the study and written informed consent was collected.

\section{Consent for publication}

None.

Competing interests

No competing interests exist. 


\section{Author details}

'School of Business, Engineering and Science, Halmstad University, Halmstad, Sweden. ${ }^{2}$ Spenshult Research and Development Center, Halmstad, Sweden. ${ }^{3}$ Department of Clinical Sciences, Lund, Section of Rheumatology, Lund University, Lund, Sweden. ${ }^{4}$ Department of Regional Health Research, University of Southern Denmark, Odense, Denmark. ${ }^{5}$ Danish Hospital for Rheumatic Diseases, University Hospital of Southern Denmark, Sønderborg, Denmark. ${ }^{6}$ Primary Health Care Unit, Department of Public Health and Community Medicine, Institute of Medicine, The Sahlgrenska Academy, University of Gothenburg, Gothenburg, Sweden.

Received: 28 April 2019 Accepted: 16 September 2019

Published online: 22 October 2019

\section{References}

1. Hoy D, March L, Brooks P, Blyth F, Woolf A, Bain C, et al. The global burden of low back pain: estimates from the global burden of disease 2010 study. Ann Rheum Dis. 2014;73(6):968-74.

2. Global Burden of Disease Study C. Global, regional, and national incidence, prevalence, and years lived with disability for 301 acute and chronic diseases and injuries in 188 countries, 1990-2013: a systematic analysis for the global burden of disease study 2013. Lancet. 2015;386(9995):743-800.

3. Hartvigsen J, Hancock MJ, Kongsted A. Louw Q. Genevay S, et al. What low back pain is and why we need to pay attention. Lancet: Ferreira ML; 2018.

4. Hoy D, Bain C, Williams G, March L, Brooks P, Blyth F, et al. A systematic review of the global prevalence of low back pain. Arthritis Rheum. 2012; 64(6):2028-37.

5. Foster NE, Thomas E, Bishop A, Dunn KM, Main CJ. Distinctiveness of psychological obstacles to recovery in low back pain patients in primary care. Pain. 2010;148(3):398-406.

6. Treede RD, Rief W, Barke A, Aziz Q, Bennett MI, Benoliel R, et al. A classification of chronic pain for ICD-11. Pain. 2015;156(6):1003-7.

7. Airaksinen O, Brox JI, Cedraschi C, Hildebrandt J, Klaber-Moffett J, Kovacs F, et al. Chapter 4. European guidelines for the management of chronic nonspecific low back pain. Eur Spine J. 2006;15 Suppl 2:S192-300.

8. Foster NE, Hill JC, Hay EM. Subgrouping patients with low back pain in primary care: are we getting any better at it? Man Ther. 2011;16(1):3-8.

9. Dunn KM, Hestbaek L, Cassidy JD. Low back pain across the life course. Best Pract Res Clin Rheumatol. 2013:27(5):591-600.

10. Hayden JA, Dunn KM, van der Windt DA, Shaw WS. What is the prognosis of back pain? Best Pract Res Clin Rheumatol. 2010;24(2):167-79.

11. Costa Lda C, Maher CG, McAuley JH, Hancock MJ, Herbert RD, Refshauge KM, et al. Prognosis for patients with chronic low back pain: inception cohort study. BMJ. 2009;339:b3829.

12. Oliveira CB, Maher CG, Pinto RZ, Traeger AC, Lin CC, Chenot JF, et al. Clinical practice guidelines for the management of non-specific low back pain in primary care: an updated overview. Eur Spine J. 2018.

13. Linton SJ. A review of psychological risk factors in back and neck pain. Spine (Phila Pa 1976). 2000;25(9):1148-56.

14. van Tulder M, Koes B, Bombardier C. Low back pain. Best Pract Res Clin Rheumatol. 2002;16(5):761-75.

15. Hill JC, Fritz JM. Psychosocial influences on low back pain, disability, and response to treatment. Phys Ther. 2011;91(5):712-21.

16. Grotle M, Foster NE, Dunn KM, Croft P. Are prognostic indicators for poor outcome different for acute and chronic low back pain consulters in primary care? Pain. 2010;151(3):790-7.

17. Buer N, Linton SJ. Fear-avoidance beliefs and catastrophizing: occurrence and risk factor in back pain and ADL in the general population. Pain. 2002; 99(3):485-91.

18. Bergman S. Management of musculoskeletal pain. Best Pract Res Clin Rheumatol. 2007:21(1):153-66.

19. Bergman S, Herrstrom P, Jacobsson LT, Petersson IF. Chronic widespread pain: a three year followup of pain distribution and risk factors. J Rheumatol. 2002;29(4):818-25.

20. Wolfe F, Smythe HA, Yunus MB, Bennett RM, Bombardier C, Goldenberg DL, et al. The American College of Rheumatology 1990 criteria for the classification of fibromyalgia. Report of the multicenter criteria committee. Arthritis Rheum. 1990;33(2):160-72.

21. Wolfe F, Clauw DJ, Fitzcharles MA, Goldenberg DL, Katz RS, Mease $P$, et al. The American College of Rheumatology preliminary diagnostic criteria for fibromyalgia and measurement of symptom severity. Arthritis Care Res (Hoboken). 2010:62(5):600-10.

22. Dean LE, Arnold L, Crofford L, Bennett R, Goldenberg D, Fitzcharles MA, et al. Impact of moving from a widespread to multisite pain definition on other fibromyalgia symptoms. Arthritis Care Res (Hoboken). 2017;69(12): 1878-86.

23. Hill JC, Dunn KM, Lewis M, Mullis R, Main CJ, Foster NE, et al. A primary care back pain screening tool: identifying patient subgroups for initial treatment. Arthritis Rheum. 2008;59(5):632-41.

24. Riis A, Rathleff MS, Jensen CE, Jensen MB. Predictive ability of the start back tool: an ancillary analysis of a low back pain trial from Danish general practice. BMC Musculoskelet Disord. 2017;18(1):360.

25. Hill JC, Whitehurst DG, Lewis M, Bryan S, Dunn KM, Foster NE, et al. Comparison of stratified primary care management for low Back pain with current best practice (STarT Back): a randomised controlled trial. Lancet. 2011;378(9802):1560-71

26. Hill JC, Dunn KM, Main CJ, Hay EM. Subgrouping low Back pain: a comparison of the STarT back tool with the Orebro musculoskeletal pain screening questionnaire. Eur J Pain. 2010;14(1):83-9.

27. Robinson HS, Dagfinrud H. Reliability and screening ability of the StarT Back screening tool in patients with low back pain in physiotherapy practice, a cohort study. BMC Musculoskelet Disord. 2017;18(1):232.

28. Ohnmeiss DD. Repeatability of pain drawings in a low back pain population. Spine (Phila Pa 1976). 2000;25(8):980-8.

29. Bergman S, Herrstrom P, Hogstrom K, Petersson IF, Svensson B, Jacobsson LT. Chronic musculoskeletal pain, prevalence rates, and sociodemographic associations in a Swedish population study. J Rheumatol. 2001;28(6):1369-77.

30. Nordstoga AL, Nilsen TIL, Vasseljen O, Unsgaard-Tondel M, Mork PJ. The influence of multisite pain and psychological comorbidity on prognosis of chronic low back pain: longitudinal data from the Norwegian HUNT study. BMJ Open. 2017;7(5):e015312.

31. Morso L, Albert H, Kent P, Manniche C, Hill J. Translation and discriminative validation of the STarT Back screening tool into Danish. Eur Spine J. 2011; 20(12):2166-73.

32. Morso L, Kent P, Albert HB, Hill JC, Kongsted A, Manniche C. The predictive and external validity of the STarT Back tool in Danish primary care. Eur Spine J. 2013;22(8):1859-67.

33. Forsbrand M, Grahn B, Hill JC, Petersson IF, Sennehed CP, Stigmar K. Comparison of the Swedish STarT Back screening tool and the short form of the Orebro musculoskeletal pain screening questionnaire in patients with acute or subacute back and neck pain. BMC Musculoskelet Disord. 2017 18(1):89.

34. Betten C, Sandell C, Hill JC, Gutke A. Cross-cultural adaptation and validation of the Swedish StarT Back screening tool. Eur J Phys. 2015;17:29-36.

35. Bergman S. Chronic musculoskeletal pain: a multifactorial process. Lund University: Lund University Publications; 2001.

36. Downie WW, Leatham PA, Rhind VM, Wright V, Branco JA, Anderson JA Studies with pain rating scales. Ann Rheum Dis. 1978;37(4):378-81.

37. Roland M, Fairbank J. The Roland-Morris disability questionnaire and the Oswestry disability questionnaire. Spine (Phila Pa 1976). 2000;25(24):3115-24

38. TheEuroQulGroup. EuroQol - a new facility for the measurement of healthRelated Quality of Life. 1990;16(3):199-208.

39. Zigmond AS, Snaith RP. The hospital anxiety and depression scale. Acta Psychiatr Scand. 1983;67(6):361-70.

40. Waddell G, Newton M, Henderson I, Somerville D, Main CI. A fear-avoidance beliefs questionnaire (FABQ) and the role of fear-avoidance beliefs in chronic low back pain and disability. Pain. 1993;52(2):157-68.

41. Hall G, Vipond N, White J. Persistent pain assessment intruments. An ACC compendium. www.acc.co.nz: Centre for Allied Health Evidence, University of South Australia; 2008

42. Kongsted A, Johannesen E, Leboeuf-Yde C. Feasibility of the STarT back screening tool in chiropractic clinics: a cross-sectional study of patients with low back pain. Chiropractic \& manual therapies. 2011;19:10.

43. Karstens S, Krug K, Hill JC, Stock C, Steinhaeuser J, Szecsenyi J, et al. Validation of the German version of the STarT-Back tool (STarT-G): a cohort study with patients from primary care practices. BMC Musculoskelet Disord. 2015;16:346

44. Page I, Abboud J. J OS, Laurencelle L, Descarreaux M. chronic low Back pain clinical outcomes present higher associations with the STarT Back screening tool than with physiologic measures: a 12-month cohort study. BMC Musculoskelet Disord. 2015;16:201. 
45. Darzi MT, Pourhadi S, Hosseinzadeh S, Ahmadi MH, Dadian M. Comparison of quality of life in low back pain patients and healthy subjects by using WHOQOL-BREF. Journal of back and musculoskeletal rehabilitation. 2014; 27(4):507-12.

46. Andersson GBJ. Epidemiological features of chronic low-back pain. Lancet. 1999;354(9178):581-5.

47. Dunn KM, Jordan K, Croft PR. Characterizing the course of low back pain: a latent class analysis. Am J Epidemiol. 2006;163(8):754-61.

\section{Publisher's Note}

Springer Nature remains neutral with regard to jurisdictional claims in published maps and institutional affiliations.

Ready to submit your research? Choose BMC and benefit from:

- fast, convenient online submission

- thorough peer review by experienced researchers in your field

- rapid publication on acceptance

- support for research data, including large and complex data types

- gold Open Access which fosters wider collaboration and increased citations

- maximum visibility for your research: over $100 \mathrm{M}$ website views per year

At BMC, research is always in progress.

Learn more biomedcentral.com/submissions 